Preprint typeset in JHEP style - HYPER VERSION

BARI-TH 2003/464

GEF-TH/2003-15

\title{
The deconfining phase transition in full QCD with two dynamical flavors
}

\author{
Paolo Cea \\ Dipartimento Interateneo di Fisica, Università di Bari and INFN - Sezione di Bari, \\ I-70126 Bari, Italy \\ E-mail: Paolo.Cea@ba.infn.it \\ Leonardo Cosmai \\ INFN - Sezione di Bari, I-70126 Bari, Italy \\ E-mail: Leonardo.Cosmai@ba.infn.it \\ Massimo D'Elia \\ Dipartimento di Fisica, Università di Genova and INFN - Sezione di Genova, \\ I-16146 Genova, Italy \\ E-mail: Massimo.Delia@ge.infn.it
}

\begin{abstract}
We investigate the deconfining phase transition in $\mathrm{SU}(3)$ pure gauge theory and in full QCD with two flavors of staggered fermions. The phase transition is detected by measuring the free energy in presence of an abelian monopole background field. In the pure gauge case our finite size scaling analysis is in agreement with the well known presence of a weak first order phase transition. In the case of 2 flavors full QCD we find, using the standard pure gauge and staggered fermion actions, that the phase transition is consistent with weak first order, contrary to the expectation of a crossover for not too large quark masses and in agreement with results obtained by the Pisa group.
\end{abstract}

Keywords: Confinement, Lattice Gauge Field Theories. 


\section{Contents}

1. Introduction 1

1.1 The lattice effective action: $T=0$

1.2 The thermal partition functional

1.3 Including fermions

1.4 The monopole free energy and the monopole condensation

2. $\mathrm{SU}(3)$ pure gauge 5

2.1 The monopole background field 5

2.2 Finite Size Scaling 8

2.3 The deconfinement temperature 11

3. QCD with two dynamical flavors 12

3.1 Numerical simulations 13

3.2 Numerical results 13

3.3 Finite Size Scaling 14

4. Conclusions 18

A. $\mathrm{U}(1)$

\section{Introduction}

Understanding QCD thermodynamics is one of the most intriguing issues of contemporary physics. Indeed the study of QCD at high temperature (and density) [1-4] is relevant for both high energy physics (e.g. ultrarelativistic heavy ion collisions) and astrophysics (compact stars). Moreover addressing QCD thermodynamics could shed light on the problem of color confinement and chiral symmetry breaking.

To detect the deconfinement phase transition in pure gauge theories the expectation value of the trace of the Polyakov loop is commonly used as an order parameter. In presence of dynamical fermions the Polyakov loop ceases to be an order parameter since $\mathrm{Z}(\mathrm{N})$ symmetry is no longer a symmetry of the action. Alternatively, in order to determine the finite temperature phase transition, one usually studies the chiral condensate, which however is not related to confinement, but to chiral symmetry breaking, and in its turn is not a good order parameter at non-zero quark masses.

A mechanism for color confinement based on dual superconductivity of the QCD vacuum by abelian monopole condensation has been proposed in [5-7]. A disorder parameter which is related to abelian monopole condensation in the dual superconductivity picture of 
confinement has been developed by the Pisa group and consists in the vacuum expectation value of a magnetically charged operator, $\langle\mu\rangle$. In Refs. [8-10] it has been shown that $\langle\mu\rangle$ is different from zero in the confined phase of $\mathrm{SU}(2)$ and $\mathrm{SU}(3)$ pure gauge theories, that it goes to zero at the deconfining phase transition, and that this is independent of the abelian projection chosen to define the magnetic charge. The same has also been verified in the case of full QCD [11].

In the present paper abelian monopole condensation is detected by looking at the free energy $[12,13]$ in presence of an abelian monopole background field, which in turn is evaluated in terms of a gauge invariant lattice effective action. This lattice effective action is defined at zero temperature by means of the lattice Schrödinger functional and at finite temperature by means of a thermal partition functional, employed so far to study the vacuum dynamics of $\mathrm{SU}(3)$ lattice gauge theory at finite temperature in presence of a constant abelian background field [14] or in presence of an abelian monopole background field $[12,13,15]$.

Since the free energy is related to the vacuum dynamics and not, like the trace of the Polyakov loop, to a symmetry of the gauge action which is washed out by the presence of dynamical fermions, we feel that it could be used to detect the finite temperature phase transition also in full QCD, as well as the order parameter $\langle\mu\rangle$ employed in Ref. [11].

In the present paper we present a finite size scaling study of the free energy in presence of an abelian monopole background field both for $\mathrm{SU}(3)$ pure gauge and two-flavor QCD.

The plan of the paper is the following. In Sections 1.1 and 1.2 we introduce the lattice effective action at zero temperature and at finite temperature. In Section 1.3 we modify the thermal partition functional for including dynamical fermions. In Section 2 we apply our method to the case of $\mathrm{SU}(3)$ pure gauge theory and, by means of a finite size scaling analysis, we find that (as expected) the deconfining phase transition is first order. In Section 3 we explore QCD thermodynamics with two staggered dynamical fermions of equal masses using the standard staggered fermion action. A finite size scaling analysis suggests that the deconfining phase transition is "weak first order", in agreement with the indications presented in $[11,16,17]$. In Section 1 we sketch our conclusions. Finally in Appendix A we report our finite size scaling investigation of the U(1) lattice gauge theory. A preliminary account of the results of the present paper appeared in Refs. [18,19].

\subsection{The lattice effective action: $T=0$}

In order to investigate vacuum structure of lattice gauge theories at zero temperature a lattice gauge invariant effective action $\Gamma\left[\vec{A}^{\text {ext }}\right]$ for an external background field $\vec{A}^{\text {ext }}$ was introduced in Refs. [20,21]. It is defined as

$$
\Gamma\left[\vec{A}^{\mathrm{ext}}\right]=-\frac{1}{L_{t}} \ln \left\{\frac{\mathcal{Z}\left[\vec{A}^{\mathrm{ext}}\right]}{\mathcal{Z}[0]}\right\} .
$$

where $L_{t}$ is the lattice size in time direction. $\vec{A}^{\text {ext }}(\vec{x})$ is the continuum gauge potential for the external static background field, the corresponding lattice links are

$$
U_{k}^{\operatorname{ext}}(\vec{x})=P \exp \int_{0}^{1} d t i a g A_{k}^{\operatorname{ext}}(\vec{x}+t a \hat{k})
$$


(a: lattice spacing, $g$ : bare gauge coupling, $P$ : path ordering operator). $\mathcal{Z}\left[\vec{A}^{\text {ext }}\right]$ is the lattice partition functional

$$
\mathcal{Z}\left[\vec{A}^{\mathrm{ext}}\right]=\int_{U_{k}\left(\vec{x}, x_{t}=0\right)=U_{k}^{\mathrm{ext}}(\vec{x})} \mathcal{D} U e^{-S_{W}},
$$

$S_{W}$ is the Wilson action and the functional integration is performed over the lattice links, but constraining the spatial links belonging to a given time slice (say $x_{t}=0$ ) to be

$$
U_{k}\left(\vec{x}, x_{t}=0\right)=U_{k}^{\mathrm{ext}}(\vec{x}), \quad(k=1,2,3),
$$

$U_{k}^{\text {ext }}(\vec{x})$ being the lattice version (see Eq. (1.2)) of the external continuum gauge field $\vec{A}^{\text {ext }}(x)=\vec{A}_{a}^{\text {ext }}(x) \lambda_{a} / 2$ (with $\lambda_{a} / 2$ Gell-Mann matrices). The temporal links are not constrained. In the case of a static background field which does not vanish at infinity we must also impose that spatial links exiting from sites belonging to the spatial boundaries (for each time slice $x_{t} \neq 0$ ) are fixed according to Eq. (1.4): in the continuum this last condition amounts to the requirement that fluctuations over the background field vanish at infinity.

The partition function defined in Eq. (1.3) is also known as lattice Schrödinger functional $[22,23]$ and in the continuum corresponds to the Feynman kernel [24,25]. Note that, at variance with the usual formulation of the lattice Schrödinger functional $[22,23]$ where a lattice cylindrical geometry is adopted, our lattice has an hypertoroidal geometry so that $S_{W}$ in Eq. (1.3) is allowed to be the standard Wilson action.

The lattice effective action $\Gamma\left[\vec{A}^{\text {ext }}\right]$ corresponds to the vacuum energy, $E_{0}\left[\vec{A}^{\text {ext }}\right]$, in presence of the background field with respect to the vacuum energy, $E_{0}[0]$, with $\vec{A}^{\mathrm{ext}}=0$

$$
\Gamma\left[\vec{A}^{\mathrm{ext}}\right] \quad \longrightarrow \quad E_{0}\left[\vec{A}^{\mathrm{ext}}\right]-E_{0}[0]
$$

The relation above is true by letting the temporal lattice size $L_{t} \rightarrow \infty$, on finite lattices this amounts to have $L_{t}$ sufficiently large to single out the ground state contribution to the energy.

Since the lattice effective action Eq. (1.1) is given in terms of the lattice Schrödinger functional, which is invariant for time-independent gauge transformation of the background field $[22,23]$, it is as well gauge invariant.

\subsection{The thermal partition functional}

If we now consider the gauge theory at finite temperature $T=1 /\left(a L_{t}\right)$ in presence of an external background field, the relevant quantity turns out to be the free energy functional defined as

$$
\mathcal{F}\left[\vec{A}^{\mathrm{ext}}\right]=-\frac{1}{L_{t}} \ln \left\{\frac{\mathcal{Z}_{\mathcal{T}}\left[\vec{A}^{\mathrm{ext}}\right]}{\mathcal{Z}_{\mathcal{T}}[0]}\right\} .
$$

$\mathcal{Z}_{\mathcal{T}}\left[\vec{A}^{\text {ext }}\right]$ is the thermal partition functional [26] in presence of the background field $\vec{A}^{\text {ext }}$, and is defined as

$$
\mathcal{Z}_{T}\left[\vec{A}^{\mathrm{ext}}\right]=\int_{U_{k}\left(\vec{x}, L_{t}\right)=U_{k}(\vec{x}, 0)=U_{k}^{\mathrm{ext}}(\vec{x})} \mathcal{D} U e^{-S_{W}},
$$


In Eq. (1.7), as in Eq. (1.3), the spatial links belonging to the time slice $x_{t}=0$ are constrained to the value of the external background field, the temporal links are not constrained. On a lattice with finite spatial extension we also usually impose that the links at the spatial boundaries are fixed according to boundary conditions Eq. (1.4), apart from the case in which the external background field vanishes at spatial infinity (as happens for the monopole field), where the choice of periodic boundary conditions in the spatial direction is equivalent to Eq. (1.4) in the thermodynamical limit. If the physical temperature is sent to zero, the thermal functional Eq. (1.7) reduces to the zero-temperature Schrödinger functional Eq. (1.3).

The free energy functional Eq. (1.6) corresponds to the free energy, $F\left[\overrightarrow{A^{\text {ext }}}\right]$, in presence of the external background field evaluated with respect to the free energy, $F[0]$, with $\overrightarrow{A^{\text {ext }}}=0$. When the physical temperature is sent to zero the free energy functional reduces to the vacuum energy functional Eq. (1.1).

\subsection{Including fermions}

When including dynamical fermions, the thermal partition functional in presence of a static external background gauge field, Eq. (1.7), becomes:

$$
\begin{aligned}
\mathcal{Z}_{T}\left[\vec{A}^{\mathrm{ext}}\right] & =\int_{U_{k}\left(L_{t}, \vec{x}\right)=U_{k}(0, \vec{x})=U_{k}^{\mathrm{ext}}(\vec{x})} \mathcal{D} U \mathcal{D} \psi \mathcal{D} \bar{\psi} e^{-\left(S_{W}+S_{F}\right)} \\
& =\int_{U_{k}\left(L_{t}, \vec{x}\right)=U_{k}(0, \vec{x})=U_{k}^{\operatorname{ext}}(\vec{x})} \mathcal{D} U e^{-S_{W}} \operatorname{det} M
\end{aligned}
$$

where $S_{W}$ is the Wilson action, $S_{F}$ is the fermionic action and $M$ is the fermionic matrix. Notice that the fermionic fields are not constrained and the integration constraint is only relative to the gauge fields: this leads, as in the usual QCD partition function, to the appearance of the gauge invariant fermionic determinant after integration on the fermionic fields. As usual we impose on fermionic fields periodic boundary conditions in the spatial directions and antiperiodic boundary conditions in the temporal direction.

\subsection{The monopole free energy and the monopole condensation}

We use the free energy functional Eq. (1.6) to evaluate the free energy in presence of an abelian monopole background field (see Sect. 2.1). If there is monopole condensation, the free energy with the monopole background field is the same as the free energy without the monopole background field $\left(\vec{A}^{\text {ext }}=0\right)$. In other words it does not cost energy to create a monopole. Therefore by evaluating the free energy by means of Eq. (1.6) we are able to detect abelian monopole condensation.

Note that, as discussed in Sect. 1.1, our free energy functional is gauge invariant for time-independent gauge transformations of the external background field. This implies that we do not need to do any gauge fixing to perform the abelian projection. Indeed, after choosing the abelian direction, needed to define the abelian monopole field through the abelian projection, due to gauge invariance of Schrödinger functional for transformations of background field, our results do not depend on the selected abelian direction, which, 
actually, can be varied by a gauge transformation. In this sense our definition is analogous to the definition given in Ref. [10], where the monopole field is defined without gauge fixing.

To evaluate the monopole free energy by means of Eq. (1.6) we should compute the ratio of two partition functions. In order to avoid the problem of dealing with partition functions, we will compute instead $[27,28]$ the derivative of the free energy $F^{\prime}(\beta)=\partial \mathcal{F} / \partial \beta$ with respect to the gauge coupling $\beta\left(\beta=6 / g^{2}\right)$. It is easy to see that $F^{\prime}(\beta)$ is given by the difference between the average plaquette $\left\langle U_{\mu \nu}>\right.$ obtained in turn from configurations with $\overrightarrow{A^{\text {ext }}}=0$ and $\vec{A}^{\text {ext }} \neq 0$

$$
F^{\prime}(\beta)=\frac{\partial \mathcal{F}(\beta)}{\partial \beta}=V\left[<U_{\mu \nu}>_{\vec{A}^{\mathrm{ext}}=0}-<U_{\mu \nu}>_{\vec{A}^{\mathrm{ext}} \neq 0}\right]
$$

where $V$ is the spatial volume. In Eq. (1.9) the dependence of the free energy functional $\mathcal{F}$ on $\beta$ (at fixed external gauge potential $\vec{A}^{\text {ext }}$ ) has been made explicit. Eventually, since $\mathcal{F}=0$ at $\beta=0$, the free energy can be obtained by numerical integration of $F^{\prime}(\beta)$

$$
F(\beta)=\int_{0}^{\beta} F^{\prime}\left(\beta^{\prime}\right) d \beta^{\prime}
$$

\section{2. $\mathrm{SU}(3)$ pure gauge}

\subsection{The monopole background field}

For $\mathrm{SU}(3)$ gauge theory the maximal abelian group is $\mathrm{U}(1) \times \mathrm{U}(1)$, therefore we may introduce two independent types of abelian monopoles using respectively the Gell-Mann matrices $\lambda_{3}$ and $\lambda_{8}$ or their linear combinations.

In the following we shall consider the abelian monopole field related to the $\lambda_{3}$ diagonal generator. In the continuum the abelian monopole field is given by

$$
g \vec{b}^{a}(\vec{x})=\delta^{a, 3} \frac{n_{\text {mon }}}{2} \frac{\vec{x} \times \vec{n}}{|\vec{x}|(|\vec{x}|-\vec{x} \cdot \vec{n})}
$$

where $\vec{n}$ is the direction of the Dirac string and, according to the Dirac quantization condition, $n_{\text {mon }}$ is an integer. The lattice links corresponding to the abelian monopole field Eq. 2.1) are (we choose $\vec{n}=\hat{x}_{3}$ )

$$
\begin{aligned}
U_{1,2}^{\operatorname{ext}}(\vec{x}) & =\left[\begin{array}{ccc}
e^{i \theta_{1,2}^{\operatorname{mon}}(\vec{x})} & 0 & 0 \\
0 & e^{-i \theta_{1,2}^{\operatorname{mon}}(\vec{x})} & 0 \\
0 & 0 & 1
\end{array}\right] \\
U_{3}^{\operatorname{ext}}(\vec{x}) & =\mathbf{1},
\end{aligned}
$$

with $\theta_{1,2}^{\text {mon }}(\vec{x})$ defined as

$$
\begin{aligned}
& \theta_{1}^{\text {mon }}(\vec{x})=-\frac{n_{\text {mon }}}{4} \frac{\left(x_{2}-X_{2}\right)}{\left|\vec{x}_{\text {mon }}\right|} \frac{1}{\left|\vec{x}_{\text {mon }}\right|-\left(x_{3}-X_{3}\right)}, \\
& \theta_{2}^{\text {mon }}(\vec{x})=+\frac{n_{\text {mon }}}{4} \frac{\left(x_{1}-X_{1}\right)}{\left|\vec{x}_{\text {mon }}\right|} \frac{1}{\left|\vec{x}_{\text {mon }}\right|-\left(x_{3}-X_{3}\right)},
\end{aligned}
$$




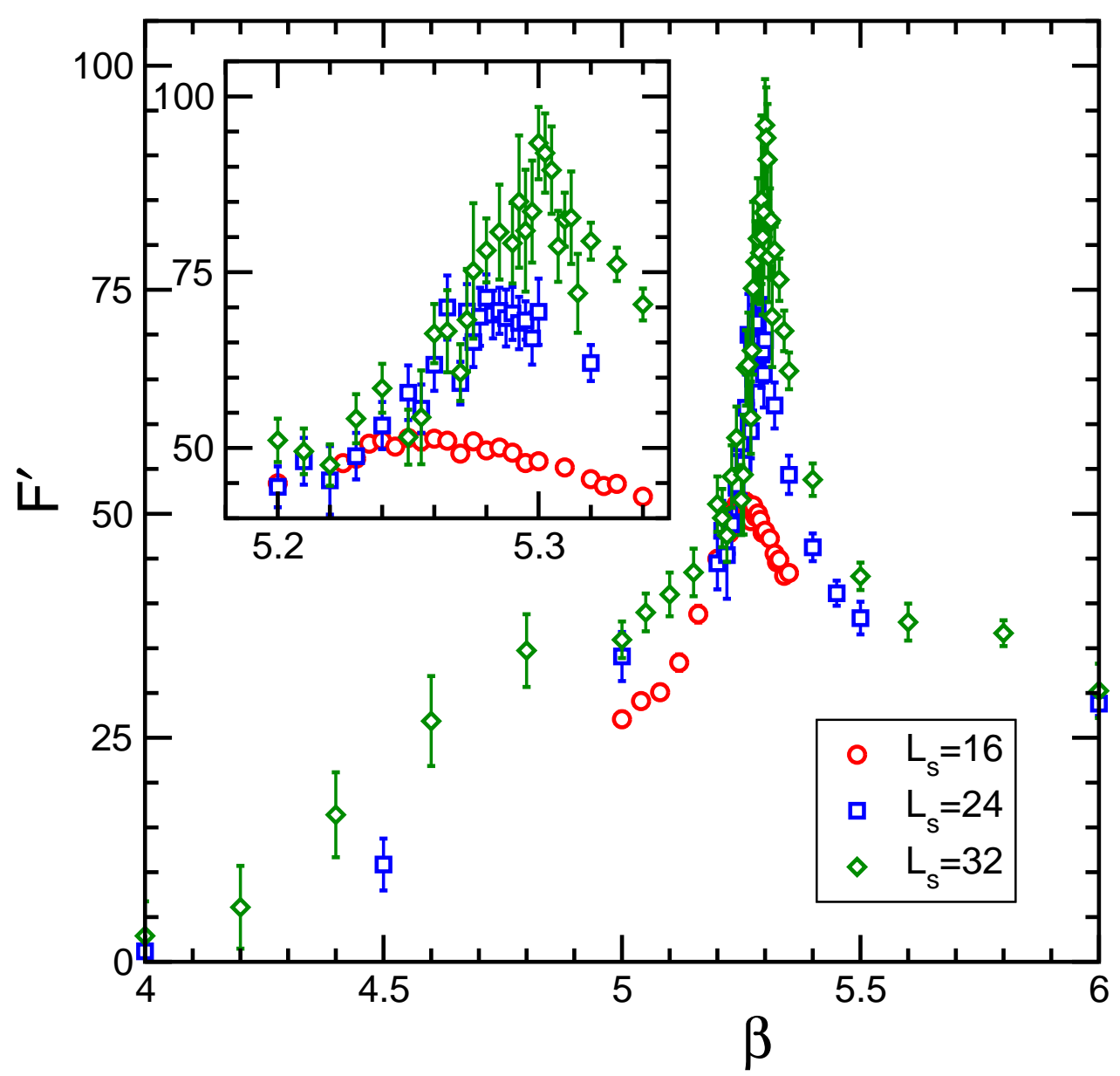

Figure 1: $F^{\prime}$ vs. $\beta\left(n_{\text {mon }}=10\right)$ for $L_{s}=16,24,32$ and $L_{t}=4$. Spatial "fixed boundary conditions" (for the significance of spatial "fixed boundary conditions" see Section 2.1).

where $\left(X_{1}, X_{2}, X_{3}\right)$ are the monopole coordinates, $\vec{x}_{\text {mon }}=(\vec{x}-\vec{X})$.

The monopole background field is introduced by constraining (see Eq. (2.2)) the spatial links exiting from the sites at the boundary of the time slice $x_{t}=0$. For what concern spatial links exiting from sites at the boundary of other time slices $\left(x_{t} \neq 0\right)$ we consider two possibilities. In the first one we constrain these links according to Eq. (2.2) (in the following we refer to this possibility as "fixed boundary conditions"). In the second one we do not impose the constraint Eq. (2.2) on the above mentioned links (this possibility will be referred as "periodic boundary conditions").

We simulate pure $\mathrm{SU}(3)$ lattice gauge theory with Wilson action. The lattice geometry is hypertoroidal. The path integral is constrained as given in Eq. (2.2). It is important to observe here that since the background field potential is not an integration variable (i.e. it does not correspond to a thermalized field) it is not necessarily subject to periodic boundary conditions.

The simulations were performed on lattices of different spatial sizes taking fixed the 


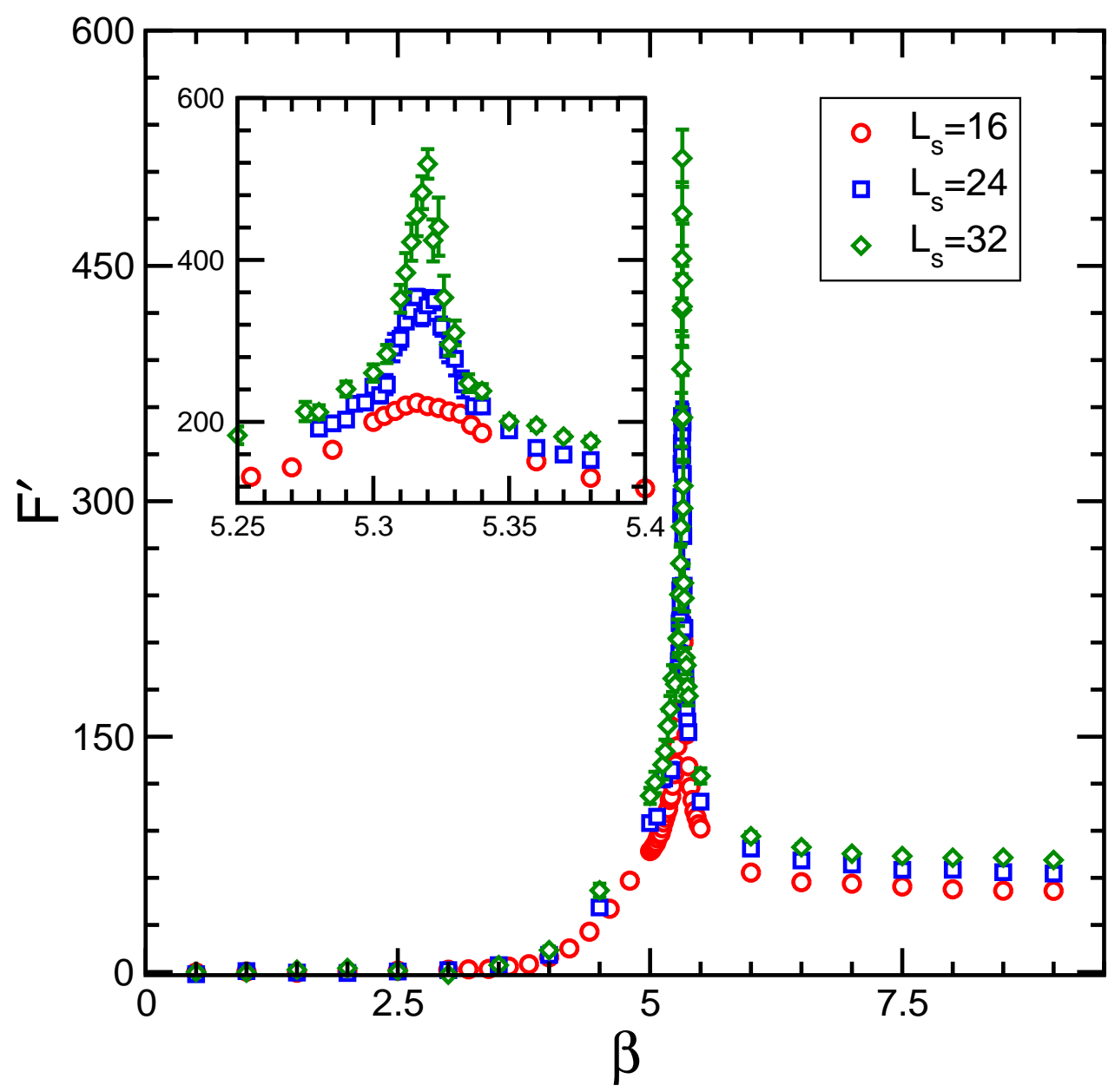

Figure 2: $F^{\prime}$ vs. $\beta\left(n_{\text {mon }}=10\right)$ for $L_{s}=16,24,32$ and $L_{t}=4$. Spatial "periodic boundary conditions" (for the significance of spatial "periodic boundary conditions" see Section 2.1).

temporal extent $\left(L_{t}=4\right)$. We consider lattices with spatial volumes $16^{3}, 24^{3}$, and $32^{3}$. Simulations were performed in part on a APE100/QH1 and in part on APEmille/crate in Bari. To upgrade SU(3) matrices we alternate a Cabibbo-Marinari heat bath sweep with one (or more) overrelaxed sweep. The statistics collected after 2,000 thermalization sweeps amounts at between 5,000 and 10,000 configurations for each value of the gauge coupling $\beta$. The statistical analysis has been done by means of jackknife resampling.

In Fig. 11 we report our numerical results for $F^{\prime}$ (Eq. (1.9)) versus $\beta$ for three different lattice sizes, $n_{\text {mon }}=10$, and spatial "fixed boundary conditions". The data display a sharp peak that increases by increasing the lattice spatial volume. The data reported in Fig. 2 refer to spatial "periodic boundary conditions" and display the same qualitative behavior, though with an increased signal in the peak region. This is to be expected, since the effective volume is larger than in the previous case with fixed spatial boundary conditions.

By inspecting Figure 2 it is evident that $F^{\prime}(\beta)=0$ in a finite range of $\beta$, starting from $\beta=0$ and below the critical coupling, signaled by a peak in $F^{\prime}(\beta)$. Therefore $F(\beta)=0$ in a finite range below the critical coupling, above which the gauge system gets deconfined. 
The vanishing of the free energy implies abelian monopole condensation.

On the other hand, $F^{\prime}(\beta)$ becomes different from zero and increases with the lattice spatial volume near the critical coupling, as expected in presence of a phase transition. Moreover in the weak coupling regime $F^{\prime}(\beta)$ stays constant and almost independent from the spatial lattice volume ${ }^{1}$. This corresponds to the classical monopole energy which depends linearly on $\beta$. It is clear that in the deconfined phase it costs a finite amount of energy to create a monopole, and so, there is not abelian monopole condensation.

In the following we will analyze the peak structure of $F^{\prime}(\beta)$ near the critical coupling and show that the data display the right finite size scaling behavior with scaling parameters compatible with a first order phase transition.

\subsection{Finite Size Scaling}

As a first step we determine the value of the critical coupling $\beta_{c}\left(L_{s}^{\mathrm{eff}}\right)$ in correspondence of each set of data (at different lattice sizes and different spatial boundary conditions). We find that our data for $F^{\prime}\left(\beta, L_{s}^{\text {eff }}\right)$ can be fitted according to

$$
F^{\prime}\left(\beta, L_{s}^{\mathrm{eff}}\right)=\frac{a\left(L_{s}^{\mathrm{eff}}\right)}{\left|\beta-\beta_{c}\left(L_{s}^{\mathrm{eff}}\right)\right|^{\alpha}}, \quad \beta<\beta_{c}\left(L_{s}^{\mathrm{eff}}\right)
$$

The quantity $L_{s}^{\mathrm{eff}}$ is defined as

$$
L_{s}^{\text {eff }}= \begin{cases}L_{s}-2 & \text { for spatial "fixed b.c.'s" } \\ L_{s} & \text { for spatial "periodic b.c.'s" }\end{cases}
$$

The definition of $L_{s}^{\text {eff }}$ given in the previous Eq. (2.5) takes into account the circumstance that for spatial "fixed boundary conditions" the effective spatial volumes is reduced since the links exiting from the sites at the spatial boundaries of each time slice $\left(x_{t} \neq 0\right)$ are constrained to the value given in Eq. (2.2).

Unlike the case of $\mathrm{U}(1)$ lattice gauge theory, discussed in Appendix $\mathrm{A}$, where $\alpha \simeq 1$, we find that our data are best fitted with $\alpha \simeq 0.35$.

In Table 1 we display $\beta_{c}\left(L_{s}^{\text {eff }}\right)$ for both spatial periodic and fixed boundary condition. From the various results for $\beta_{c}\left(L_{s}^{\mathrm{eff}}\right)$ we are able to get an estimate of $\beta_{c} \equiv \beta_{c}\left(L_{s}^{\mathrm{eff}}=\infty\right)$. We try the following fit

$$
\beta_{c}\left(L_{s}^{\mathrm{eff}}\right)=\beta_{c}+d_{1}\left(L_{s}^{\mathrm{eff}}\right)^{-1 / \nu} .
$$

We find a rather good fit $\left(\chi^{2} /\right.$ d.o.f. $\left.\simeq 0.8\right)$ with $d_{1} \simeq 0.6$ and

$$
\beta_{c}=5.3228 \pm 0.0024, \quad \nu=0.334 \pm 0.021
$$

The critical exponent $\nu$ is consistent with a first order phase transition where $1 / \nu=3$.

\footnotetext{
${ }^{1}$ We note a difference with the Pisa order parameter $\mu$ : in that case $\frac{d}{d \beta} \ln \langle\mu\rangle$ increases linearly with the spatial volume in the weak coupling limit, since $\mu$ is a magnetically charged operator subject to magnetic charge superselection in the deconfined phase [29].
} 


\begin{tabular}{|lcc|}
\hline \hline & spatial "fixed boundary conditions" & (see Section 2.1) \\
\hline$L_{s}$ & $L_{s}^{\text {eff }}$ & $\beta_{c}$ \\
\hline 16 & 14 & $5.4033(959)$ \\
24 & 22 & $5.2976(247)$ \\
32 & 30 & $5.3138(108)$ \\
\hline \hline \multicolumn{3}{|c|}{ spatial "periodic boundary conditions" } \\
\hline$L_{s}$ & $L_{s}^{\text {eff }}$ & $\beta_{c}$ \\
\hline 16 & 16 & $5.3296(33)$ \\
24 & 24 & $5.3200(56)$ \\
32 & 32 & $5.3199(100)$ \\
\hline \hline
\end{tabular}

Table 1: The value of $\beta_{c}$ in correspondence of different lattice sizes and different spatial boundary conditions, obtained by fitting Eq. (2.4) to the lattice data for $F^{\prime}\left(\beta, L_{s}^{\text {eff }}\right)$ (see Eq. (1.9)).

The behavior of $F^{\prime}\left(\beta, L_{s}^{\mathrm{eff}}\right)$ in the critical region can be investigated by using finite size scaling techniques. Equations (2.4) and (2.6) suggest the following scaling law

$$
F^{\prime}\left(\beta, L_{s}^{\mathrm{eff}}\right)=\frac{a_{1}}{\left|\beta-\beta_{c}-d_{1}\left(L_{s}^{\mathrm{eff}}\right)^{-1 / \nu}\right|^{\alpha}}=\frac{a_{1}\left(L_{s}^{\mathrm{eff}}\right)^{\alpha / \nu}}{\left|\left(L_{s}^{\mathrm{eff}}\right)^{1 / \nu}\left(\beta-\beta_{c}\right)-d_{1}\right|^{\alpha}},
$$

so that $F^{\prime}\left(\beta, L_{s}^{\text {eff }}\right) /\left(L_{s}^{\text {eff }}\right)^{\alpha / \nu}$ is a universal function of the scaling variable

$$
x=\left(L_{s}^{\mathrm{eff}}\right)^{1 / \nu}\left(\beta-\beta_{c}\right) .
$$

Note that Eq. (2.8) gives a sensible result in the thermodynamical limit $L_{s}^{\mathrm{eff}} \rightarrow \infty$

$$
F^{\prime}(\beta)=\frac{a_{1}}{\left|\beta-\beta_{c}\right|^{\alpha}}, \quad \beta<\beta_{c}
$$

while $F^{\prime}\left(\beta=\beta_{c}, L_{s}^{\text {eff }}\right)$ diverges like $\left(L_{s}^{\text {eff }}\right)^{\alpha / \nu}$ when $L_{s}^{\text {eff }} \rightarrow \infty$.

Accordingly we fitted our lattice data with the scaling law

$$
F^{\prime}\left(\beta, L_{s}^{\mathrm{eff}}\right)=\frac{a_{1}\left(L_{s}^{\mathrm{eff}}\right)^{\gamma}}{\left|\left(L_{s}^{\mathrm{eff}}\right)^{1 / \nu}\left(\beta-\beta_{c}\right)-d_{1}\right|^{\alpha}}
$$

where we expect that $\gamma=\alpha / \nu$.

The output of the fits are reported in Table 2. The parameter $d_{1}$ has been fixed at the value obtained with the fit Eq. (2.6). We see clearly that $\alpha / \nu$ agrees with $\gamma$ within statistical errors, as expected if we want a sensible result in the thermodynamical limit $L_{s}^{\mathrm{eff}} \rightarrow \infty$ (see Eq. 2.11) ).

In Figs. 1 , 司 we plot $F^{\prime}\left(\beta, L_{s}^{\mathrm{eff}}\right)$ rescaled with the factor $1 /\left(L_{s}^{\mathrm{eff}}\right)^{\gamma}$ versus the scaling variable $x$ defined in Eq. (2.9), respectively for spatial "fixed boundary conditions" and for spatial "periodic boundary conditions". The full line is

$$
\frac{F^{\prime}\left(\beta, L_{s}^{\mathrm{eff}}\right)}{\left(L_{s}^{\mathrm{eff}}\right)^{\gamma}}=\frac{a_{1}}{\left|\left(L_{s}^{\mathrm{eff}}\right)^{1 / \nu}\left(\beta-\beta_{c}\right)-d_{1}\right|^{\alpha}} .
$$




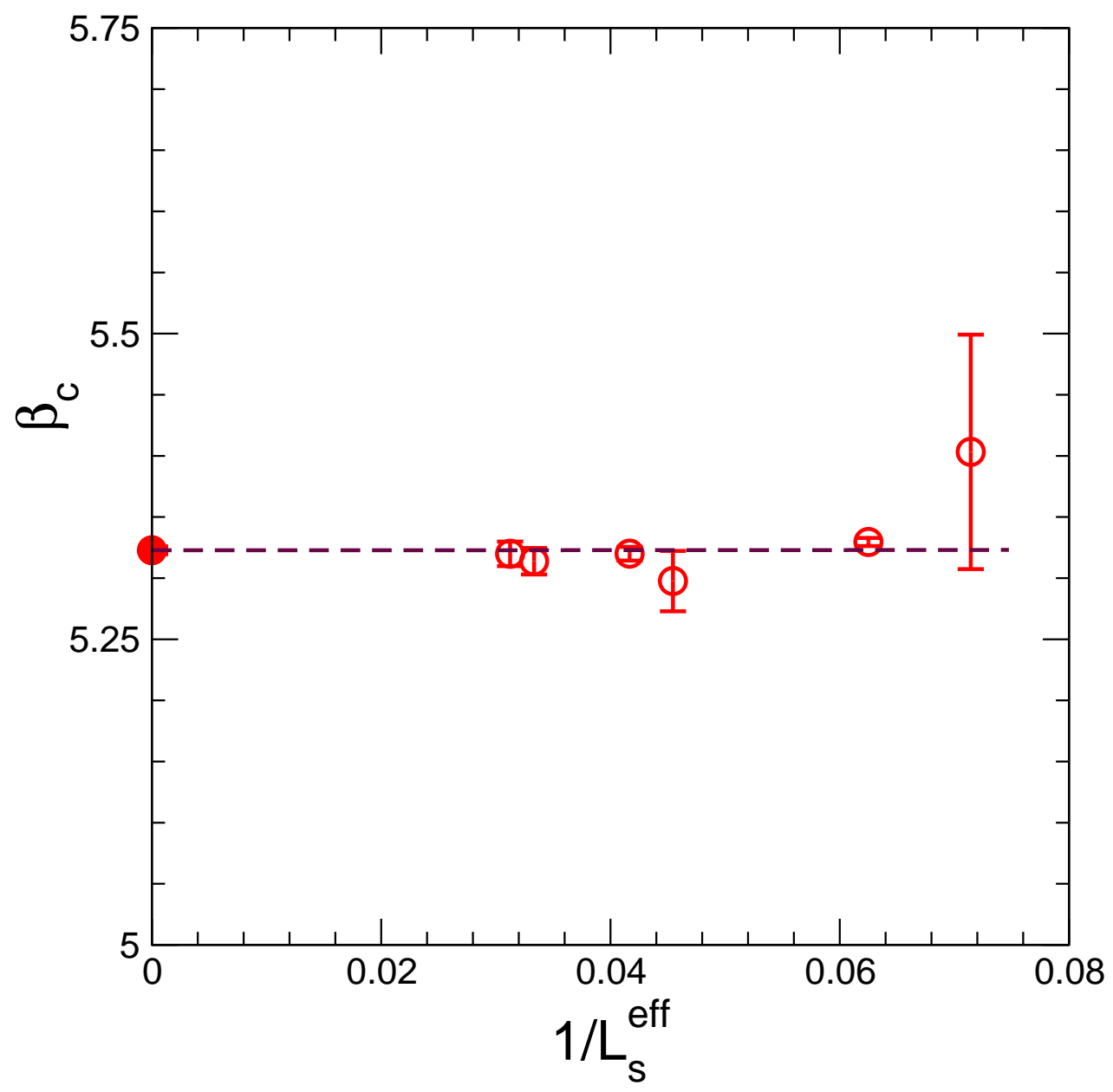

Figure 3: The values of $\beta_{c}\left(L_{s}^{\mathrm{eff}}\right)$ (open circles), versus $1 / L_{s}^{\mathrm{eff}}$, obtained at different values of $L_{s}^{\text {eff }}$

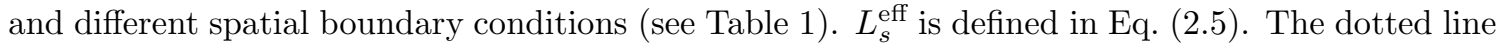
is the fit Eq. (2.6). The value $\beta_{c}\left(L_{s}^{\mathrm{eff}}=\infty\right)$ is also plotted (full circle).

We find that the scaling relation holds quite well for a very large range of $x$. The quality of the scaling can be inferred looking at Figs. 1 and 5. Moreover looking at Table 2 we remarkably see that the critical parameters $\alpha, \beta_{c}, \nu$, and $\gamma$ agree for both sets of data.

Finally in Fig. 6 we display $F^{\prime}\left(\beta, L_{s}^{\text {eff }}\right) /\left(L_{s}^{\text {eff }}\right)^{\gamma}$ versus the scaling variable $x$ for both periodic and fixed spatial boundary conditions. We see that the numerical data nicely display the same scaling behavior.

It is interesting to comment on the behavior of $\exp (-F(\beta) / T)$, which is the analogous of disorder parameter of Refs. [8-10], in the thermodynamical limit implied by Eq. (2.10). Indeed we have that

$$
\exp \left(-\frac{F(\beta)}{T}\right)=\exp \left(-\frac{1}{T} \int_{\beta_{0}}^{\beta} F^{\prime}\left(\beta^{\prime}\right) d \beta^{\prime}\right) \quad, \quad \beta_{0}<\beta<\beta_{c}
$$

while we already know that $\exp (-F(\beta) / T)=1$ for $\beta<\beta_{0}$ irrespective of the lattice size 


\begin{tabular}{|c|c|c|c|c|c|}
\hline \multicolumn{6}{|c|}{ spatial "fixed boundary conditions" (see Section 2.1) } \\
\hline$a_{1}$ & $\gamma$ & $\bar{\beta}$ & $\nu$ & $d_{1}$ & $\bar{\alpha}$ \\
\hline 12.199 & 1.247 & 5.3251 & 0.335 & 0.6 & 0.351 \\
\hline \pm 3.9004 & \pm 0.089 & \pm 0.0110 & \pm 0.026 & constant & \pm 0.035 \\
\hline \multicolumn{6}{|c|}{ spatial "periodic boundary conditions" (see Section 2.1) } \\
\hline$a_{1}$ & $\gamma$ & $\beta_{c}$ & $\nu$ & $d_{1}$ & $\alpha$ \\
\hline 13.461 & 1.510 & 5.3222 & 0.340 & 0.6 & 0.347 \\
\hline \pm 1.337 & \pm 0.555 & \pm 0.0013 & \pm 0.020 & constant & \pm 0.009 \\
\hline
\end{tabular}

Table 2: The values of the parameters obtained by fitting Eq. (2.11) to the data for the derivative of the monopole free energy Eq. (1.9) on lattices with spatial volumes $16^{3}, 24^{3}$, and $32^{3}$ and spatial "fixed" or "periodic" boundary conditions respectively.

(see Fig. (2)). From Eq. (2.10) we get

$$
\exp \left(-\frac{F(\beta)}{T}\right)=\exp \left[-\frac{1}{T} \frac{a_{1}}{1-\alpha}\left(\left|\beta_{0}-\beta_{c}\right|^{1-\alpha}-\left|\beta-\beta_{c}\right|^{1-\alpha}\right)\right], \quad \beta_{0}<\beta<\beta_{c} .
$$

So that $F(\beta)$ decreases when $\beta \rightarrow \beta_{c}$ if $0<\alpha<1$ tending to a finite value at $\beta=\beta_{c}$. On the other hand, for $\alpha=1$ it is easy to see that $\exp (-F(\beta) / T)$ decreases to zero as a power of $\left(\beta_{c}-\beta\right)$. Thus we conclude that for $\alpha<1$ we have a discontinuous jump of $\exp (-F(\beta) / T)$ at $\beta_{c}$ and the strength of the discontinuity weakens when $\alpha \rightarrow 1$. However, it must be stressed that the discontinuous jump of $\exp (-F(\beta) / T)$ is exceedingly small so that $\exp (-F(\beta) / T)$ decreases almost continuously toward zero when $\beta \rightarrow \beta_{c}$.

\subsection{The deconfinement temperature}

Before ending the discussion of pure $\mathrm{SU}(3)$ gauge theory, we would like to remark that using the data for $F^{\prime}(\beta)$ we are able to get an estimate of the continuum extrapolated critical temperature $T_{c}$ in units of the square root of the string tension $\sqrt{\sigma}$. We will show here that our estimate is consistent, even though with a quite large error, with the updated value in the literature.

In fact, by fitting Eq. (2.4) to our data for $F^{\prime}(\beta)$ obtained on lattices $32^{3} \times L_{t}\left(L_{t}=\right.$ $6,7,8$, with spatial periodic boundary conditions), we are able to get an estimate of the critical coupling $\beta_{c}\left(L_{t}\right)$ corresponding to each $L_{t}$. To fix a physical scale we consider the string tension $a \sqrt{\sigma}$ at each value of $\beta_{c}\left(L_{t}\right)$. The string tension is obtained on a symmetric lattice with the Wilson action. To this purpose we use the data for the string tension as parameterized in Eq.(4.4) of Ref. [30].

In Fig. 7 our data for $T_{c} / \sqrt{\sigma}$ are displayed versus $a T_{c}$. The continuum extrapolation using an ansatz quadratic in $a T_{c}$ gives the estimate $T_{c} / \sqrt{\sigma}=0.635 \pm 0.147$ to be confronted with the average estimate in the literature $T_{c} / \sqrt{\sigma}=0.640 \pm 0.015$ (see Table 3 of Ref. [31]).

Our conclusion is that our method allows to get an estimate of $T_{c} / \sqrt{\sigma}$ although the statistical uncertainty is quite large, mainly due to a large error in the evaluation of $\beta_{c}\left(L_{t}=\right.$ 6). A better estimate of $\beta_{c}$ and then of $T_{c} / \sqrt{\sigma}$ could be achieved by means of the density spectral method. 


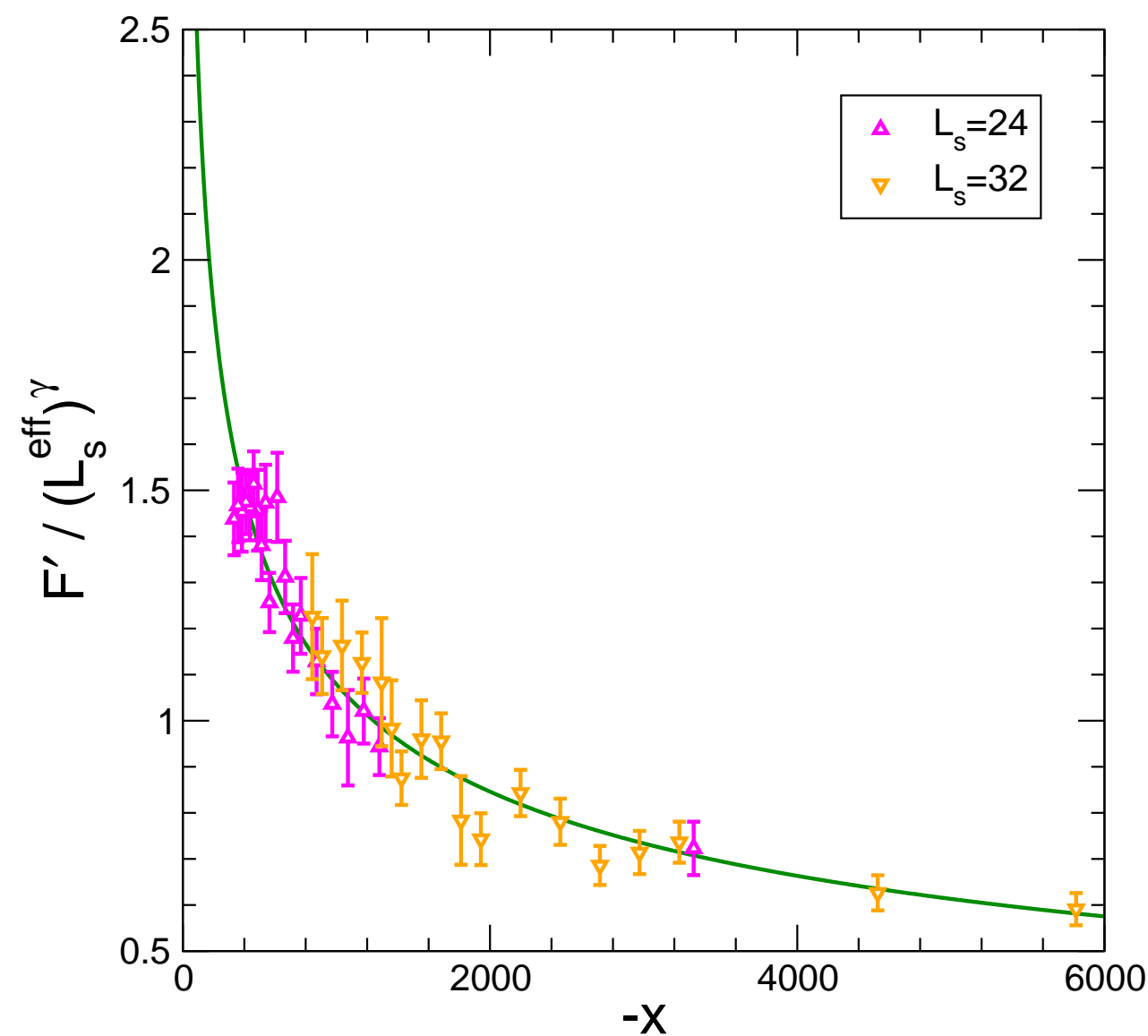

Figure 4: The derivative of the monopole free energy with respect to the gauge coupling $\beta$ (Eq. (1.9)) rescaled with $\left(L_{s}^{\text {eff }}\right)^{\gamma}$ versus the scaling variable $x$ (Eq. (2.9)). Data refer to simulations with spatial "fixed boundary conditions". The full line is the scaling curve Eq. (2.12) with the parameters from Table日.

\section{QCD with two dynamical flavors}

We will account for results achieved from the study of QCD with two dynamical fermions using the standard staggered fermion action. As discussed in the Introduction, we simulate the theory with a "cold" time-slice (say $x_{t}=0$ ) where the spatial links are constrained to be a (lattice) abelian monopole background field (Eq. (2.2)). According to Section 2.1 the spatial links exiting from the sites belonging to the boundaries of the $x_{t} \neq 0$ temporal slices are also constrained according to Eq. (2.2): we have not considered the possibility of spatial periodic boundary conditions in this case.

We compute the derivative of the monopole background field free energy with respect to the gauge coupling, as given in Eq. (1.9), where now the expectation value is evaluated with the full QCD action (details and numerical results in Sections 3.1 and 3.2). Our main goal is to try to use our data for $F^{\prime}(\beta)$ on different spatial volumes and different bare quark masses to infer the critical behavior of two flavors full QCD near the deconfining transition. 


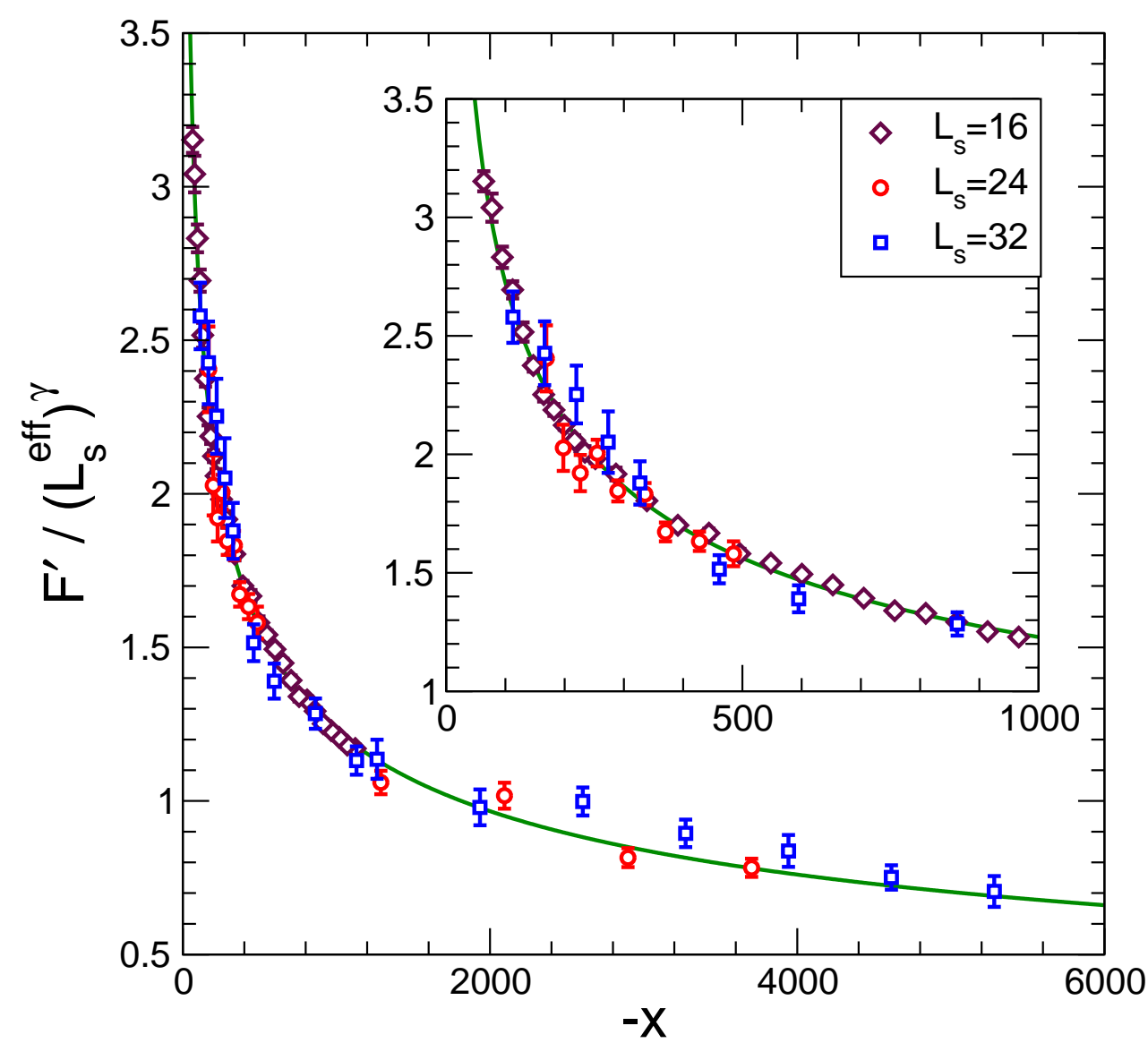

Figure 5: The derivative of the monopole free energy with respect to the gauge coupling $\beta$ (Eq. (1.9)) rescaled with $\left(L_{s}^{\text {eff }}\right)^{\gamma}$ versus the scaling variable $x$ (Eq. (2.9)). Data refer to simulations with spatial "periodic boundary conditions". The full line is the scaling curve Eq. (2.12) with the parameters from Table日.

\subsection{Numerical simulations}

We used a slight modification of the standard HMC R-algorithm [32] for two degenerate flavors of staggered fermions with quark mass $m_{q}$ : the links which are frozen are not evolved during the molecular dynamics trajectory and the corresponding conjugate momenta are set to zero. We have collected about 2000 thermalized trajectories for each value of $\beta$ at $L_{s}=16,20$ and about 1000 thermalized trajectories for each value of $\beta$ at $L_{s}=32$. Each trajectory consists of 125 molecular dynamics steps and has total length 1 . The computer simulations have been performed on the APEmille crate.

\subsection{Numerical results}

In Fig. 8 we compare $F^{\prime}(\beta)$ for two staggered degenerate flavors with quark mass $m_{q}=$ 0.075 and in the quenched case. In the 2 flavors full QCD case the signal in the peak region gets enhanced with respect to the quenched case and the position of the peak shifts to a smaller value of $\beta$. 


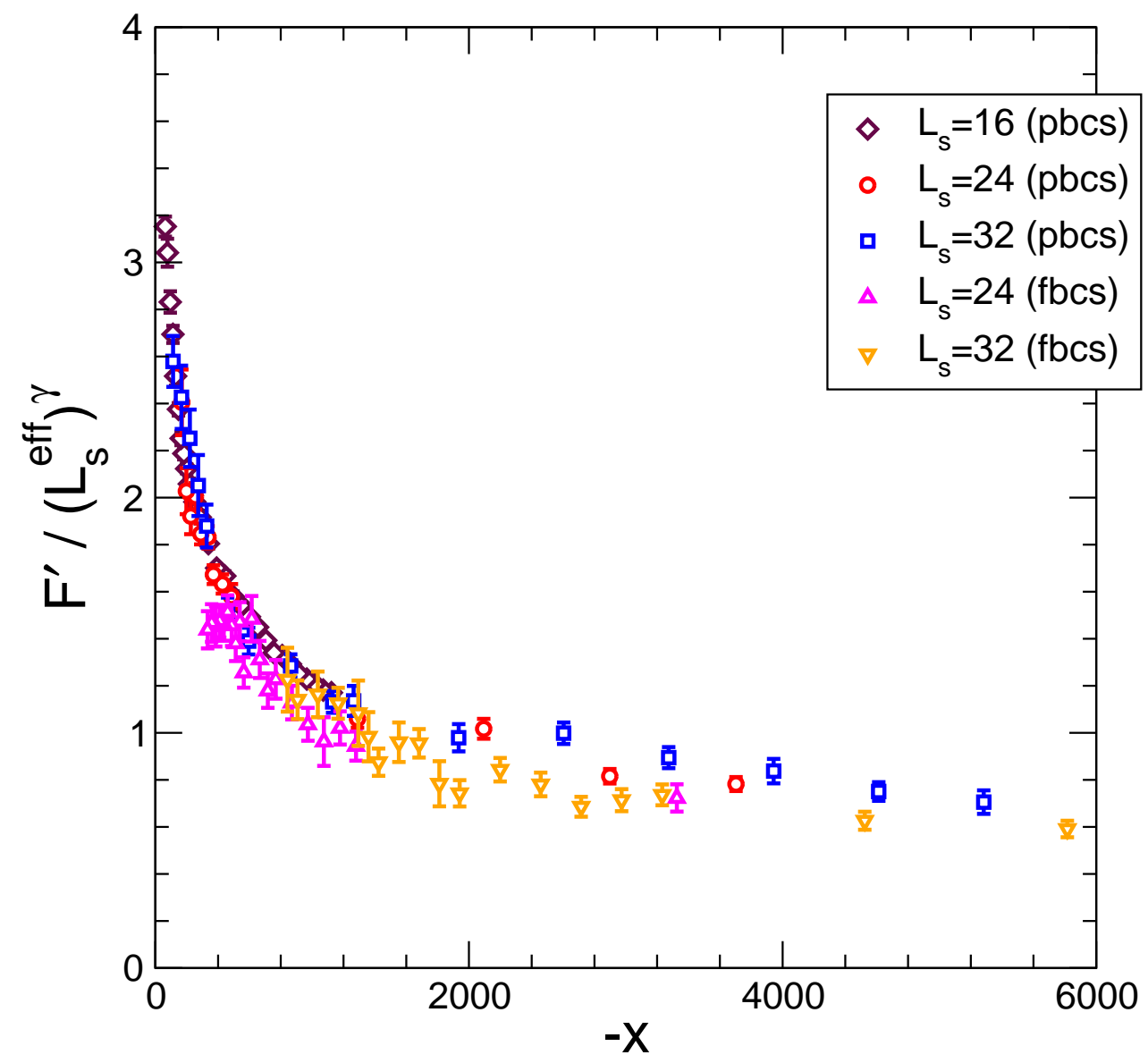

Figure 6: We plot together data from Fig. 目(fbcs) and Fig. 目(pbcs).

In Fig. 9 we display $F^{\prime}(\beta)$ in the case of 2 dynamical flavors $\left(m_{q}=0.075,16^{3} \times 4\right.$ lattice) compared with the chiral condensate $\langle\psi \bar{\psi}\rangle$. Data displayed in Fig. 9 suggest that the peak in $F^{\prime}(\beta)$ corresponds to the drop of the chiral condensate. We varied the lattice size $L_{s}\left(L_{s}=16,20,32\right)$ and the staggered quark mass $m_{q}\left(m_{q}=0.075,0.2676,0.5003\right)$. At fixed $m_{q}$, as in the quenched case, we find that the sharp peak increases with the lattice spatial volume. Moreover at fixed spatial volume the critical coupling depends on $m_{q}$.

\subsection{Finite Size Scaling}

We would like to use the numerical data collected at different lattice size and staggered quark mass to perform a finite size scaling analysis. According to Eq. (2.8) we try the scaling law

$$
F^{\prime}\left(\beta, L_{s}^{\mathrm{eff}}\right)=\frac{a_{1}\left(L_{s}^{\mathrm{eff}}\right)^{\gamma}}{\left|\left(L_{s}^{\mathrm{eff}}\right)^{1 / \nu}\left(\beta-\beta_{c}\left(m_{q}\right)\right)-d_{1}\right|^{\alpha}},
$$

where the critical coupling $\beta_{c}\left(m_{q}\right)$ depends on the quark mass $m_{q}$. The dependence of the critical coupling $\beta_{c}\left(m_{q}\right)$ on the quark mass is determined by the chiral critical point [33-35]. In the thermodynamical limit, by known universality arguments the critical couplings will 


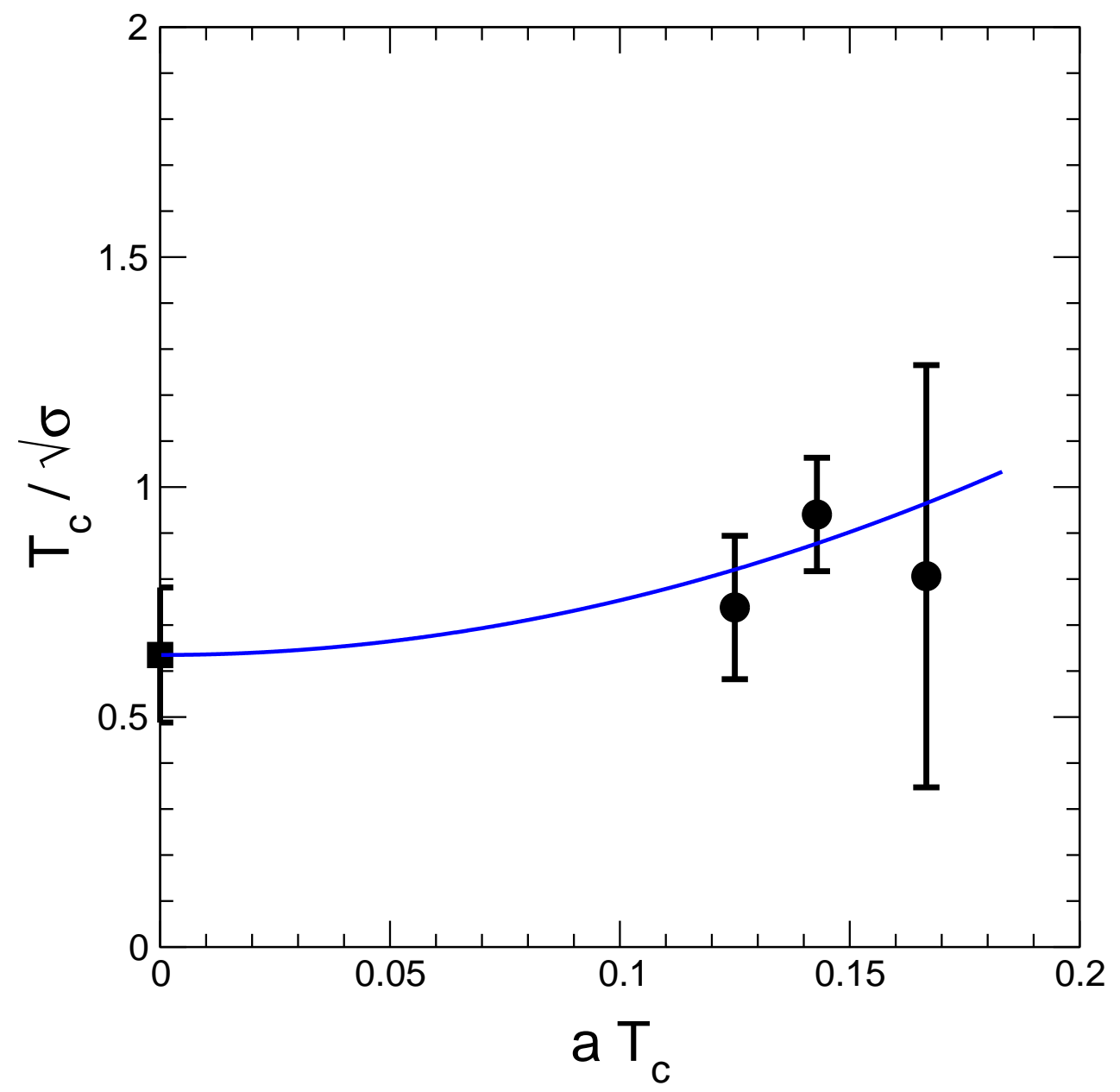

Figure 7: Continuum extrapolation for $T_{c} / \sqrt{\sigma}$ (full circles). The extrapolated value is $T_{c} / \sqrt{\sigma}=$ $0.635 \pm 0.147$ (full square).

scale like

$$
\beta_{c}\left(m_{q}\right)=\beta_{c}\left(m_{q}=0\right)+c m_{q}^{1 / \beta \delta},
$$

where $1 / \beta \delta$ is a combination of critical exponents which for the case of 2-flavors QCD are expected to be those of the three-dimensional $\mathrm{O}(4)$ symmetric spin models:

$$
\frac{1}{\beta \delta} \simeq 0.5415 \text {. }
$$

Inserting Eq. (3.2) into Eq. (3.1) we are lead to the following scaling law

$$
F^{\prime}\left(\beta, L_{s}^{\mathrm{eff}}, m_{q}\right)=\frac{a_{1}\left(L_{s}^{\mathrm{eff}}\right)^{\gamma}}{\left|\left(L_{s}^{\mathrm{eff}}\right)^{1 / \nu}\left(\beta-\beta_{c}(0)-c m_{q}^{\eta}\right)-d_{1}\right|^{\alpha}},
$$

where again $\gamma=\alpha / \nu$ assures a sensible thermodynamical limit. Note that, to take care of finite volume effects, the exponent $\eta$ is expected to be:

$$
\eta=\frac{\nu_{c}}{\nu}, \quad \nu_{c}=\frac{\nu^{\prime}}{\beta \delta},
$$




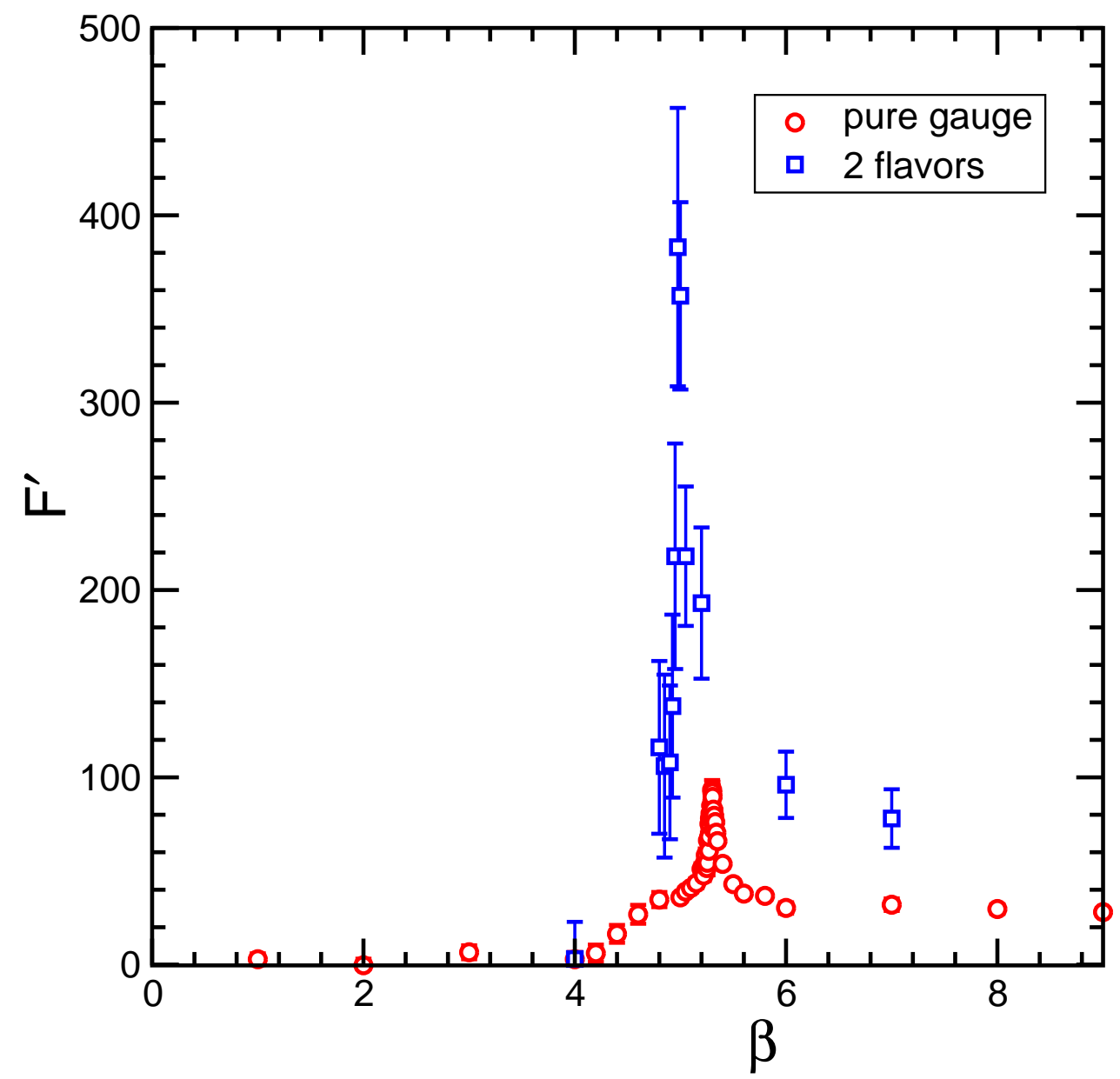

Figure 8: $F^{\prime}$ vs. $\beta$ in the case of pure $\mathrm{SU}(3)$ gauge theory (open circles) and full QCD with 2 dynamical flavors (open squares). Lattice size is $32^{3} \times 4$.

where $\nu^{\prime}, \beta$, and $\delta$ are the chiral critical exponents.

Indeed, Eqs. (3.4) and (3.5) assure that in the scaling region

$$
\frac{F^{\prime}\left(\beta, L_{s}^{\mathrm{eff}}, m_{q}\right)}{\left(L_{s}^{\mathrm{eff}}\right)^{\gamma}}=\Phi\left(\left(L_{s}^{\mathrm{eff}}\right)^{1 / \nu}\left(\beta-\beta_{c}(0)\right),\left(L_{s}^{\mathrm{eff}}\right)^{1 / \nu_{c}} m_{q}\right)
$$

In our case the relevant chiral critical exponents are those of the three-dimensional $\mathrm{O}(4)$ symmetric spin models where [34]

$$
\nu^{\prime}=0.7423, \quad \nu_{c}=0.4019 .
$$

In Table 3 we report the results obtained by fitting Eq. (3.4) to all our lattice data.

From Table 3 we can see that $\alpha / \nu=2.35 \pm 0.34$ consistent with $\gamma=2.00 \pm 0.47$ (see Section 2.2). Concerning the parameter $\eta$ we find that it is poorly determined by our data. If we constrain $\eta$ in our fit we get $\eta=1.10 \pm 0.19$ which, together with $\nu=0.31 \pm 0.03$ leads to (see Eq. (3.5)) $\nu_{c}=0.34 \pm 0.07$ consistent with the value reported in Eq. (3.7). However if we release the constraint on $\eta$ our data can also be fitted with smaller values 


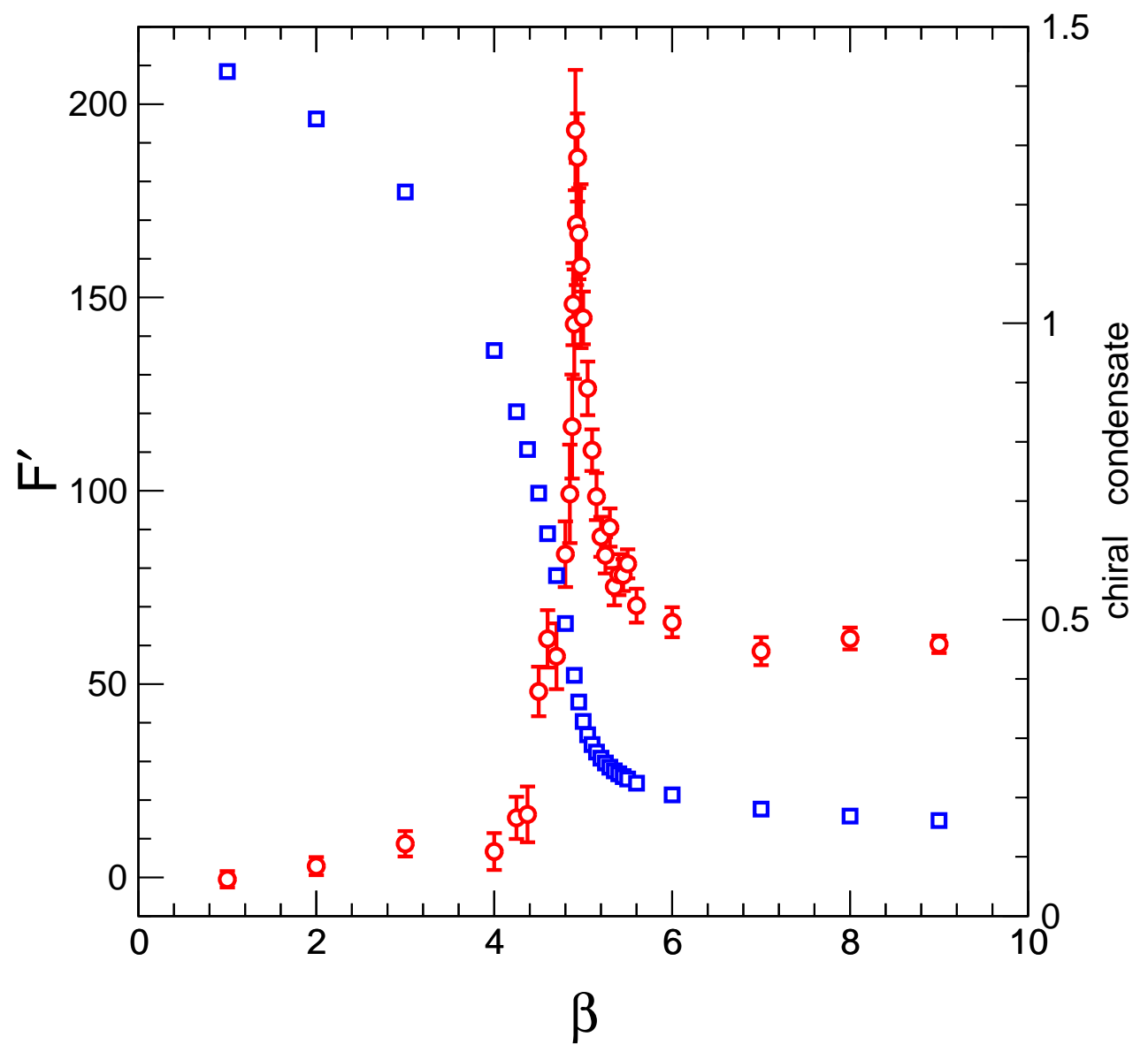

Figure 9: $F^{\prime}$ on a $16^{3} \times 4$ lattice in the case of full QCD with 2 dynamical flavors, is displayed together with chiral condensate.

\begin{tabular}{|llllllll|}
\hline \hline \multicolumn{7}{|c|}{ spatial "fixed boundary conditions" } \\
\hline$a_{1}$ & $\gamma$ & $\beta_{c}(0)$ & $c$ & $\eta$ & $\nu$ & $d_{1}$ & $\alpha$ \\
\hline 79.4 & 2.00 & 4.9933 & 0.54 & 1.10 & 0.31 & 0.6 & 0.728 \\
\pm 76.6 & \pm 0.47 & \pm 0.0138 & \pm 0.11 & \pm 0.19 & \pm 0.03 & constant & \pm 0.078 \\
\hline \hline
\end{tabular}

Table 3: The values of the parameters obtained by fitting Eq. (3.4) to the data for the derivative of the monopole free energy Eq. (1.9) in two-flavors full QCD on lattices with spatial volumes $16^{3}$, $24^{3}$, and $32^{3}$ and $L_{t}=4$.

for $\eta$ without altering significantly the other parameters. Moreover by confronting the exponent $\alpha$ in Table 3 with the corresponding value for the $\mathrm{SU}(3)$ pure gauge in Table 2 we conclude that our data for full QCD with two dynamical flavors are compatible with a first order phase transition $(\nu=0.31 \pm 0.03)$ but, in the sense discussed at the end of Section 2.2, this is weaker than in the quenched case. Our results are in agreement with the indications for a first order phase transition in full QCD with 2 dynamical flavours (in the same range of quark masses) obtained in Refs. [11,16,17]. 


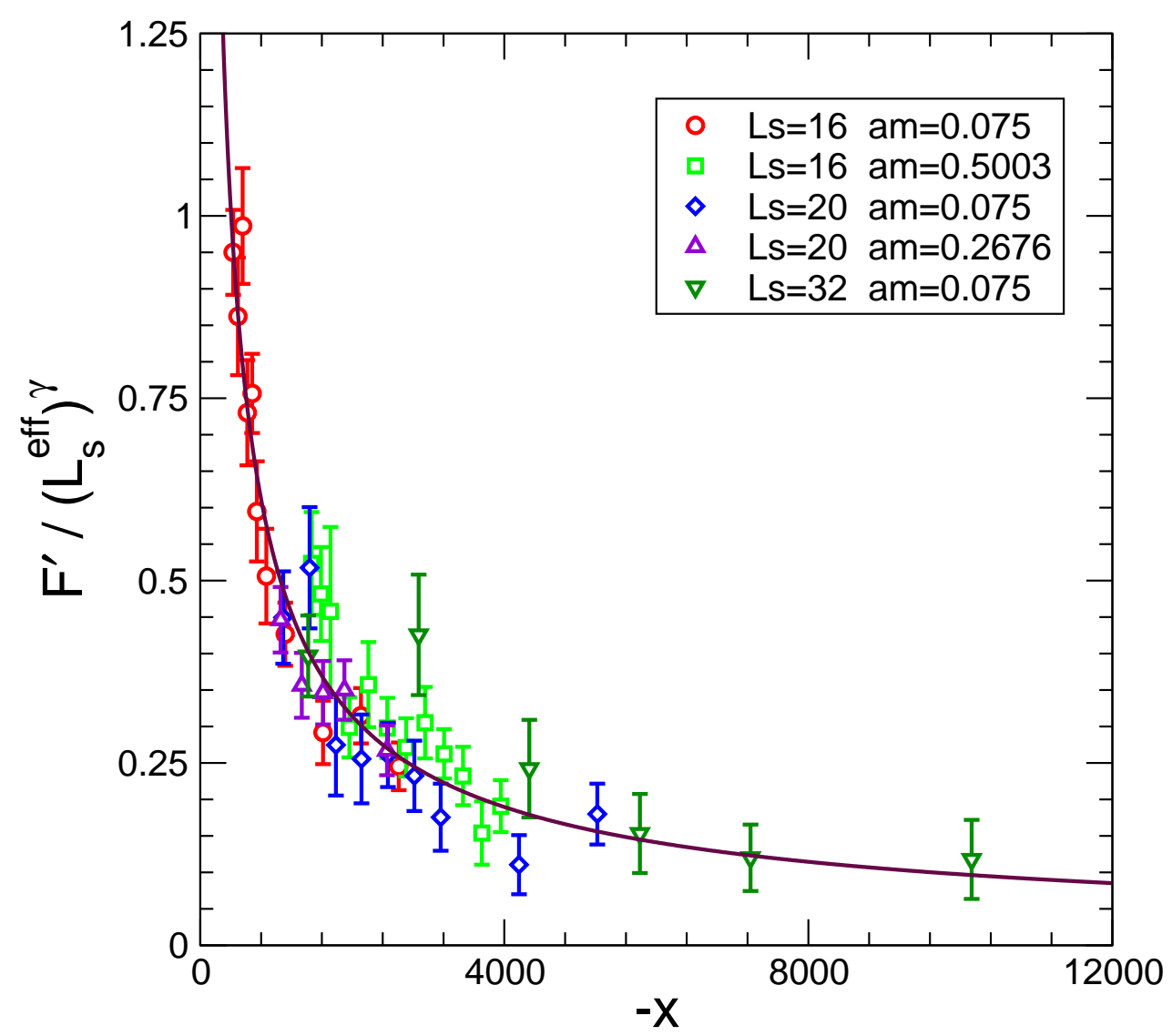

Figure 10: $F^{\prime}\left(\beta, L_{s}^{\mathrm{eff}}, m_{q}\right)$ rescaled by the factor $\left(L_{s}^{\mathrm{eff}}\right)^{\gamma}$. The values of $L_{s}^{\mathrm{eff}}$ and $m_{q}=a m$ are displayed in the legend.

\section{Conclusions}

Let us conclude by stressing the main results of this paper. We investigated the nature of deconfining phase transition in $\mathrm{SU}(3)$ pure gauge theory and in full QCD with two flavors of staggered fermions. To locate the phase transition we used the derivative of the monopole free energy with respect to the gauge coupling. The monopole free energy is defined by means of a gauge invariant thermal partition functional in presence of the abelian monopole background field. In the pure gauge case our finite size scaling analysis indicate a weak first order phase transition. We get also an estimate of $T_{c} / \sqrt{\sigma}$ extrapolated to the continuum in good agreement with updated value in the literature. Moreover our method has been checked in $\mathrm{U}(1)$ pure gauge theory giving results in agreement with previous investigations which we present in Appendix A.

In the case of 2 flavors full QCD, we performed simulations by varying spatial lattice sizes and quark masses. We find that deconfinement transition in full QCD with 2 degenerate dynamical flavors is consistent with a weak first order phase transition, contrary to the expectation of a crossover for not too large quark masses, but in agreement with the indications obtained with Refs. $[11,16,17]$. Our results deserve further investigations that we plan to do in the near future. In particular we would like to stress that we have used the 


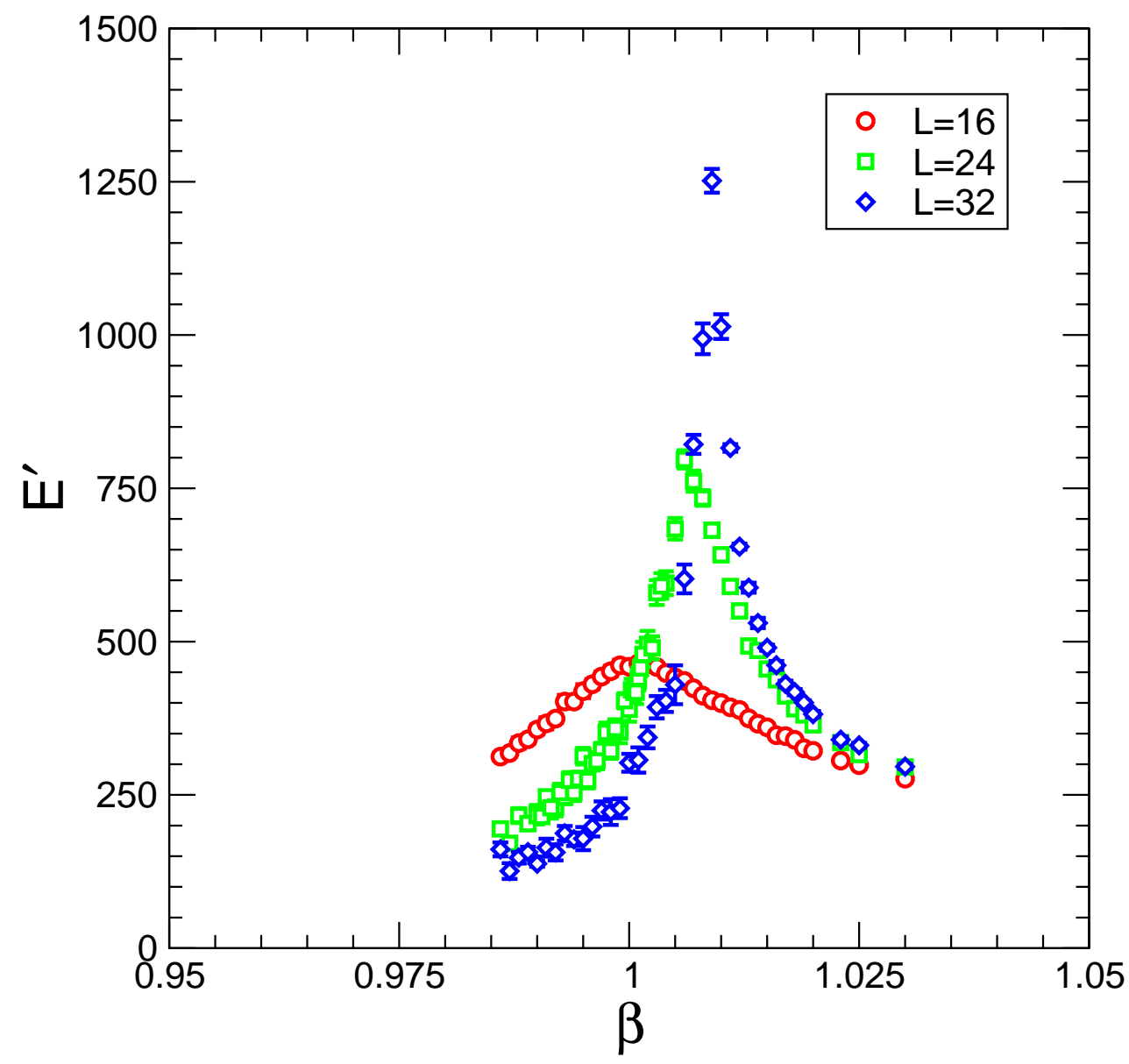

Figure 11: U(1) lattice gauge theory. The derivative with respect to $\beta$ of the monopole energy on lattices $L^{4}(L=16,24,32)$ and $n_{\text {mon }}=20$.

standard pure gauge and staggered fermion action with $L_{t}=4$, where finite lattice spacing scaling violations could still be important, so that we plan to make use of an improved action and/or of a larger value of $L_{t}$. We plan to investigate the critical region by means of the density spectral method. However, it is worthwhile to stress that, if this result will be confirmed the phase diagram of QCD with dynamical flavors should be reconsidered.

\section{Acknowledgments}

We greatly appreciate useful discussions with Adriano Di Giacomo.

\section{A. $\mathrm{U}(1)$}

In this Appendix we take into account pure gauge $\mathrm{U}(1)$ lattice theory at zero physical temperature, essentially for the purpose of showing that using our method the known results of compact $\mathrm{U}(1)$ are reproduced. We consider $\mathrm{U}(1)$ lattice gauge theory in a monopole background field. In the continuum the magnetic monopole field with the Dirac string in 


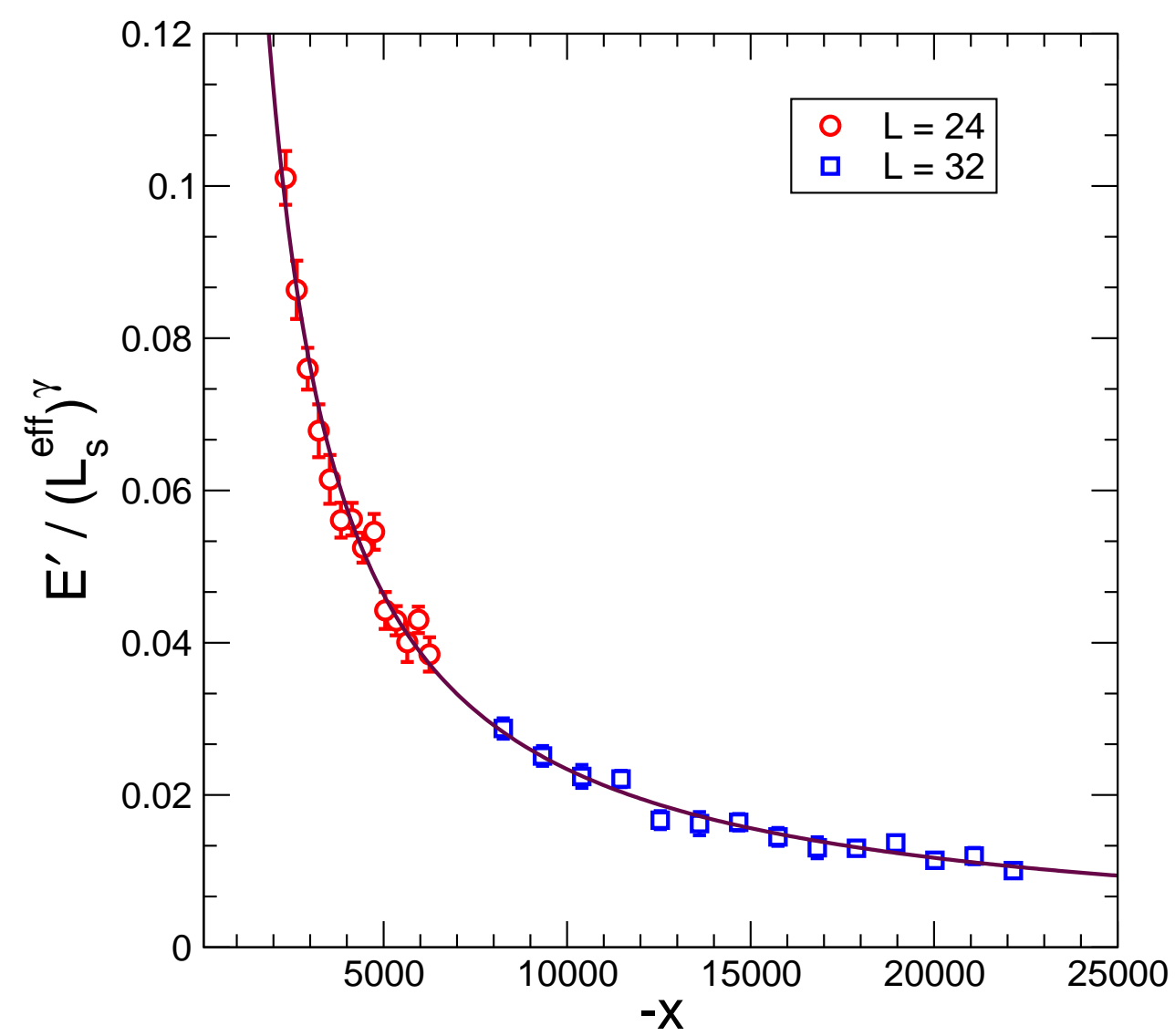

Figure 12: U(1) lattice gauge theory. $E^{\prime}(\beta)$ rescaled by $\left(L_{s}^{\text {eff }}\right)^{\gamma}$ on lattices $24^{4}$ and $32^{4}$. Solid line is the best fit according to the scaling law Eq. (2.8).

the direction $\vec{n}$ is

$$
e \vec{b}^{a}(\vec{x})=\frac{n_{\text {mon }}}{2} \frac{\vec{x} \times \vec{n}}{|\vec{x}|(|\vec{x}|-\vec{x} \cdot \vec{n})},
$$

where, according to the Dirac quantization condition, $n_{\text {mon }}$ is an integer and $e$ is the electric charge (magnetic charge $=n_{\text {mon }} / 2 e$ ). On the lattice the links belonging to the $x_{t}=0$ timeslice and to the spatial boundaries of the $x_{t} \neq 0$ time-slices are constrained as (we choose $\left.\vec{n}=\hat{x}_{3}\right)$

$$
\begin{aligned}
& U_{1,2}^{\operatorname{ext}}(\vec{x})=\cos \left[\theta_{1,2}^{\operatorname{mon}}(\vec{x})\right]+i \sin \left[\theta_{1,2}^{\operatorname{mon}}(\vec{x})\right] \\
& U_{3}^{\operatorname{ext}}(\vec{x})=\mathbf{1}
\end{aligned}
$$

with

$$
\begin{aligned}
& \theta_{1}^{\text {mon }}(\vec{x})=-\frac{n_{\text {mon }}}{2} \frac{\left(x_{2}-X_{2}\right)}{\left|\vec{x}_{\text {mon }}\right|} \frac{1}{\left|\vec{x}_{\text {mon }}\right|-\left(x_{3}-X_{3}\right)}, \\
& \theta_{2}^{\text {mon }}(\vec{x})=+\frac{n_{\text {mon }}}{2} \frac{\left(x_{1}-X_{1}\right)}{\left|\vec{x}_{\text {mon }}\right|} \frac{1}{\left|\vec{x}_{\text {mon }}\right|-\left(x_{3}-X_{3}\right)} .
\end{aligned}
$$

In Equation A.3) $\left(X_{1}, X_{2}, X_{3}\right)$ are the monopole coordinates and $\vec{x}_{\text {mon }}=(\vec{x}-\vec{X})$. In the numerical simulations we put the lattice Dirac monopole at the center of the time slice 
$x_{t}=0$. To avoid the singularity due to the Dirac string we locate the monopole between two neighboring sites.

The abelian monopole condensation is detected by means of $E^{\prime}(\beta)$, the derivative of the vacuum energy (with respect to the gauge coupling) in presence of the monopole background field and is given by (see Sect. 1.1)

$$
E=-\frac{1}{L_{t}} \frac{\mathcal{Z}\left[\vec{A}^{\mathrm{ext}}\right]}{\mathcal{Z}[0]}
$$

where $\mathcal{Z}\left[\vec{A}^{\text {ext }}\right]$ is the lattice Schrödinger functional with $\vec{A}^{\text {ext }}$ the monopole background field and, according to the physical interpretation of the effective action, we denote $\Gamma$ in Eq. (1.1) with $E$.

Again, to avoid the problem of evaluating partition functions, we compute $E^{\prime}(\beta)$, the derivative with respect to $\beta$ of the monopole energy $E(\beta)$, defined in terms of the lattice effective action Eq. (1.1). $E^{\prime}(\beta)$ is computed by the analogous of Eq. (1.9).

In Fig. 11 the data for $E^{\prime}(\beta)$, with $n_{\text {mon }}=10$, on lattices $L^{4}(L=16,24,32)$ are displayed. We observe a sharp peak near the deconfining transition $(\beta \simeq 1.01)$. To extract quantitative features from the lattice data we do a finite size scaling analysis. To this purpose we employ the scaling law Eq. (2.11) to fit the data for $E^{\prime}(\beta)$ on lattices $24^{4}$ and $32^{4}$. The best-fit parameters are reported in Table 4 . In Fig. 12 we report $E^{\prime}\left(\beta, L_{s}^{\text {eff }}\right)$, rescaled by $1 /\left(L_{s}^{\text {eff }}\right)^{\gamma}$, versus the scaling variable $\left(L_{s}^{\text {eff }}\right)^{1 / \nu}\left(\beta-\beta_{c}\right)$. As we can see the scaling law Eq. (2.11) holds very well for a wide range of the scaling variable $x$. By inspecting

\begin{tabular}{|llllll|}
\hline \hline \multicolumn{5}{c|}{ spatial "fixed boundary conditions" } & (see Section 2.1) \\
\hline$a_{1}$ & $\gamma$ & $\beta_{c}$ & $\nu$ & $d_{1}$ & $\alpha$ \\
\hline 285.2 & 2.80 & 1.0107 & 0.245 & 200.0 & 1.019 \\
\pm 102.0 & \pm 0.70 & \pm 0.0010 & \pm 0.010 & 102.8 & \pm 0.036 \\
\hline \hline
\end{tabular}

Table 4: The values of the parameters obtained by fitting Eq. (2.8) to the data for the derivative of the monopole energy Eq. (1.9) on lattices with volumes $24^{4}$, and $32^{4}$.

Table 1 we can see that $\alpha / \nu=4.16 \pm 0.22$ which is consistent with $\gamma+1=3.8 \pm 0.7$. Indeed, according to Eq. (A.4), to obtain a sensible result for $\beta<\beta_{c}$ in the thermodynamical limit, we must have $\alpha / \nu=\gamma+1$. Indeed, we see that this last relation is satisfied within statistical uncertainty. Our results are in good quantitative agreement with previous finite size scaling study of the disorder parameter performed in Ref. [36]. Moreover our determination of the critical coupling $\beta_{c}$ is consistent with the most recent determination reported in the literature [37]. For what concern the order of phase transition our data indicate a weak first order phase transition. In fact the parameter $\nu$ is consistent with $1 / d$ for $d=4$. Moreover $\alpha \simeq 1$ within errors indicating that the analogous of the disorder parameter of Refs. [8-10] (see the discussion at the end of Sect. 2.2) goes to zero almost continuously as $\beta \rightarrow \beta_{c}$. In this sense, we can say that the first order deconfinement transition in $\mathrm{U}(1)$ is weaker than in $\mathrm{SU}(3)$. 


\section{References}

[1] H. Satz, Phase transitions in QCD, Nucl. Phys. A681 (2001) 3-21, hep-ph/0007209.

[2] F. Karsch, Lattice QCD at high temperature and density, Lect. Notes Phys. 583 (2002) 209-249, hep-lat/0106019.

[3] E. Laermann and O. Philipsen, Status of lattice QCD at finite temperature, hep-ph/0303042,

[4] D. H. Rischke, The quark-gluon plasma in equilibrium, nucl-th/0305030.

[5] G. 't Hooft, The confinement phenomenon in quantum field theory, in High Energy Physics, EPS International Conference, Palermo, 1975.

[6] S. Mandelstam, Vortices and quark confinement in non Abelian gauge theories, Phys. Rept. 23 (1976) 245.

[7] G. Parisi, Quark imprisonment and vacuum repulsion, Phys. Rev. D11 (1975) 970.

[8] A. Di Giacomo, B. Lucini, L. Montesi, and G. Paffuti, Colour confinement and dual superconductivity of the vacuum. i, Phys. Rev. D61 (2000) 034503, hep-lat/9906024.

[9] A. Di Giacomo, B. Lucini, L. Montesi, and G. Paffuti, Colour confinement and dual superconductivity of the vacuum. ii, Phys. Rev. D61 (2000) 034504, hep-lat/9906025.

[10] J. M. Carmona, M. D'Elia, A. Di Giacomo, B. Lucini, and G. Paffuti, Color confinement and dual superconductivity of the vacuum. iii, Phys. Rev. D64 (2001) 114507, hep-lat/0103005.

[11] J. M. Carmona, M. D'Elia, L. Del Debbio, A. Di Giacomo, B. Lucini, and G. Paffuti, Color confinement and dual superconductivity in full QCD, Phys. Rev. D66 (2002) 011503, hep-lat/0205025.

[12] P. Cea and L. Cosmai, Gauge invariant study of the monopole condensation in non Abelian lattice gauge theories, Phys. Rev. D62 (2000) 094510, hep-lat/0006007.

[13] P. Cea and L. Cosmai, Abelian monopole and vortex condensation in lattice gauge theories, JHEP 11 (2001) 064.

[14] P. Cea and L. Cosmai, Abelian chromomagnetic fields and confinement, JHEP 02 (2003) 031, hep-lat/0204023.

[15] P. Cea and L. Cosmai, Magnetic condensation and confinement in lattice gauge theory, Nucl. Phys. Proc. Suppl. 94 (2001) 486-489, hep-lat/0010034.

[16] J. M. Carmona, M. D’Elia, L. Del Debbio, A. Di Giacomo, B. Lucini, G. Paffuti, and C. Pica, Deconfining transition in full QCD, Nucl. Phys. Proc. Suppl. 119 (2003) 697-699, hep-lat/0209082.

[17] J. M. Carmona, M. D’Elia, L. Del Debbio, A. Di Giacomo, B. Lucini, G. Paffuti, and C. Pica, Deconfining transition in two-flavor QCD, hep-lat/0309035.

[18] P. Cea, L. Cosmai, and M. D'Elia, Monopole condensation in full QCD using the Schrödinger functional, Nucl. Phys. Proc. Suppl. 119 (2003) 748-750, hep-lat/0209118.

[19] P. Cea, L. Cosmai, and M. D'Elia, Investigations on the deconfining phase transition in QCD, hep-lat/0309031.

[20] P. Cea, L. Cosmai, and A. D. Polosa, The lattice Schrödinger functional and the background field effective action, Phys. Lett. B392 (1997) 177-181, hep-lat/9601010. 
[21] P. Cea and L. Cosmai, Probing the non-perturbative dynamics of SU(2) vacuum, Phys. Rev. D60 (1999) 094506, hep-lat/9903005.

[22] M. Lüscher, R. Narayanan, P. Weisz, and U. Wolff, The Schrödinger functional: A renormalizable probe for non Abelian gauge theories, Nucl. Phys. B384 (1992) 168-228, hep-lat/9207009.

[23] M. Lüscher and P. Weisz, Background field technique and renormalization in lattice gauge theory, Nucl. Phys. B452 (1995) 213-233, hep-lat/9504006.

[24] G. C. Rossi and M. Testa, The structure of Yang-Mills theories in the temporal gauge. 1. General formulation, Nucl. Phys. B163 (1980) 109.

[25] G. C. Rossi and M. Testa, The structure of Yang-Mills theories in the temporal gauge. 2. Perturbation theory, Nucl. Phys. B176 (1980) 477.

[26] D. J. Gross, R. D. Pisarski, and L. G. Yaffe, $Q C D$ and instantons at finite temperature, Rev. Mod. Phys. 53 (1981) 43.

[27] A. Hasenfratz, P. Hasenfratz, and F. Niedermayer, Electric fluxes and twisted free energies in SU(3), Nucl. Phys. B329 (1990) 739.

[28] L. Del Debbio, A. Di Giacomo, and G. Paffuti, Detecting dual superconductivity in the ground state of gauge theory, Phys. Lett. B349 (1995) 513-518, hep-lat/9403013.

[29] M. D'Elia, A. Di Giacomo, and B. Lucini, Magnetic charge superselection in the deconfined phase of Yang-Mills theory, hep-lat/0309004.

[30] R. G. Edwards, U. M. Heller, and T. R. Klassen, Accurate scale determinations for the Wilson gauge action, Nucl. Phys. B517 (1998) 377-392, hep-lat/9711003.

[31] M. J. Teper, Glueball masses and other physical properties of $S U(N)$ gauge theories in $D=$ 3+1: a review of lattice results for theorists, hep-th/9812187.

[32] S. Gottlieb, W. Liu, D. Toussaint, R. L. Renken, and R. L. Sugar, Hybrid molecular dynamics algorithms for the numerical simulation of quantum chromodynamics, Phys. Rev. D35 (1987) 2531-2542.

[33] F. Karsch and E. Laermann, Susceptibilities, the specific heat and a cumulant in two flavor QCD, Phys. Rev. D50 (1994) 6954-6962, hep-lat/9406008.

[34] J. Engels, S. Holtmann, T. Mendes, and T. Schulze, Finite-size-scaling functions for $3 d$ O(4) and O(2) spin models and QCD, Phys. Lett. B514 (2001) 299-308, hep-lat/0105028.

[35] F. Karsch, E. Laermann, and A. Peikert, Quark mass and flavor dependence of the QCD phase transition, Nucl. Phys. B605 (2001) 579-599, hep-lat/0012023.

[36] A. Di Giacomo and G. Paffuti, A disorder parameter for dual superconductivity in gauge theories, Phys. Rev. D56 (1997) 6816-6823, hep-lat/9707003.

[37] G. Arnold, B. Bunk, T. Lippert, and K. Schilling, Compact QED under scrutiny: It's first order, Nucl. Phys. Proc. Suppl. 119 (2003) 864-866, hep-lat/0210010. 